\title{
BMJ Open Risk factors of falls in inpatients and their practical use in identifying high-risk persons at admission: Fukushima Medical University Hospital cohort study
}

\author{
Takehito Hayakawa, ${ }^{1}$ Shigeatsu Hashimoto, ${ }^{2}$ Hideyuki Kanda, ${ }^{3}$ Noriko Hirano, ${ }^{2}$ \\ Yumi Kurihara, ${ }^{2}$ Takako Kawashima, ${ }^{2}$ Tetsuhito Fukushima ${ }^{1}$
}

To cite: Hayakawa T, Hashimoto S, Kanda $\mathrm{H}$, et al. Risk factors of falls in inpatients and their practical use in identifying high-risk persons at admission: Fukushima Medical University Hospital cohort study. BMJ Open 2014;4:e005385. doi:10.1136/bmjopen-2014005385

- Prepublication history for this paper is available online. To view these files please visit the journal online (http://dx.doi.org/10.1136/ bmjopen-2014-005385).

Received 4 April 2014 Revised 30 June 2014 Accepted 10 July 2014

CrossMark

For numbered affiliations see end of article.

Correspondence to Takehito Hayakawa; thayaka@fmu.ac.jp

\section{ABSTRACT}

Objectives: To clarify the risk factors for falls in hospital settings and to propose the use of such factors to identify high-risk persons at admission. Design: Prospective cohort study.

Setting: Fukushima Medical University Hospital, Japan, from August 2008 and September 2009.

Participants: 9957 adult consecutive inpatients admitted to our hospital.

Methods: Information was collected at admission from clinical records obtained from a structured questionnaire conducted in face-to-face interviews with subjects by nurses and doctors and fall events were collected from clinical records.

Results: The proportion of patients who fell during follow-up was $2.5 \%$ and the incidence of falls was 3.28 per 100 person-days. There were significant differences in age, history of falling, cognitive dysfunction, planned surgery, wheelchair use, need for help to move, use of a remote caring system, rehabilitation, use of laxative, hypnotic or psychotropic medications and need for help with activities of daily living (ADL) between patients who did and did not fall. Multivariable adjusted ORs for falls showed that age, history of falls and need for help with ADL were common risk factors in both men and women. Using psychotropic medication also increased the risk of falling in men while cognitive dysfunction and use of hypnotic medication increased the risk of falling in women. Planned surgery was associated with a low risk of falls in women.

Conclusions: To prevent falls in inpatients it is important to identify high-risk persons. Age, history of falling and the need for help with ADL are the most important pieces of information to be obtained at admission. Care plans for patients including fall prevention should be clear and considered.

\section{INTRODUCTION}

Injuries resulting from falls are a major problems in elderly people, representing one of

\section{Strengths and limitations of this study}

- This study clarifies the risk factors for falling in the hospital setting.

- The study lacks disease type, but it is important to evaluate preventive factors against falls.

- This is an important cohort study of risk factors for falling in consecutive inpatients in Japan.

the main causes of longstanding pain, functional impairment, disability and death. ${ }^{1}$ The incidence rate of falls in inpatients is three times that for community-dwelling persons aged $\geq 65$ years. $^{2}$ A meta-analysis found a significantly increased risk of falls from psychotropic medication, antiarrhythmic medications, digoxin and diuretics, suggesting that healthcare interventions are an important risk factor for falls in hospitals. ${ }^{3}$ The prevention of falls and injuries is not easy because they are complex events caused by a combination of intrinsic impairments and disabilities. In the clinical setting, an effective intervention to prevent falls involves assessing and addressing an individual's risk factors for falling, and an individualised approach is recommended in the American and British Geriatric Societies' practice guidelines. ${ }^{4}$ A review of randomised controlled trials of fall prevention interventions concluded that several types of intervention were effective, including training in strength and balance, modification of hazards and withdrawal of psychotropic medication. ${ }^{5}$

There have been few cohort studies of the risk factors for falls in elderly patients in the hospital setting. The aim of this study was to clarify the risk factors for falls in inpatients by cohort and to propose the use of such factors to identify high-risk persons at admission. 
Table 1 Incidence of falls per 100 person-days, Fukushima Medical University Hospital cohort

\begin{tabular}{|c|c|c|c|c|}
\hline \multirow[b]{2}{*}{$\begin{array}{l}\text { Age } \\
\text { category }\end{array}$} & \multicolumn{2}{|l|}{ Men } & \multicolumn{2}{|c|}{ Women } \\
\hline & $\mathbf{N}$ & $\begin{array}{l}\text { Per } 100 \\
\text { person-days }\end{array}$ & $\mathbf{N}$ & $\begin{array}{l}\text { Per } 100 \\
\text { person-days }\end{array}$ \\
\hline 50 & 953 & 1.14 & 1548 & 1.45 \\
\hline $50-64$ & 1362 & 1.65 & 1176 & 1.38 \\
\hline $65-74$ & 1398 & 2.85 & 1042 & 3.83 \\
\hline 75 & 1035 & 4.33 & 955 & 5.36 \\
\hline
\end{tabular}

\section{METHODS}

A cohort study was carried out in Fukushima Medical University Hospital, Fukushima, Japan. The subjects comprised 9957 adult consecutive inpatients admitted to our hospital between August 2008 and September 2009 who were followed until discharge. Informed consent to participate in the study was obtained from inpatients on admission to the hospital.

Information was collected from clinical records at admission obtained from a structured questionnaire conducted in the form of a face-to-face interview with subjects by nurses and doctors. The information obtained included age, sex, history of falling, history of diseases (eg, cognitive dysfunction), reason for hospitalisation (eg, surgery planned), need for care or help (eg, wheelchair use, help to move, use of a remote caring system, rehabilitation) and medication (eg, laxative, sedative, hypnotic, psychotropic). Participants were asked whether each of seven activities of daily living (ADL) (standing, sitting, dressing, eating, toileting, evacuation and washing their face) could be accomplished with or without help. The need for help with any one of the seven activities was defined as a low level of ADL. Subjects were scored on the Manual Muscle Test (MMT),${ }^{6}$ where impairment was defined as MMT $<4$.

The Statistical Package for Social Sciences (SPSS Japan V.14.0J, Tokyo, Japan) was used for analyses. Baseline characteristics were compared between those with and those without falls during the follow-up period. Differences were assessed using the $\chi^{2}$ test for bivariate analysis. Means for continuous values were analysed using analysis of variance. A multivariate adjusted OR of falls according to sex was calculated using logistic regression models adjusted for age, history of falling, cognitive dysfunction, planned surgery, wheelchair use, need for help to move, use of a remote caring system, rehabilitation, use of laxative, hypnotic or psychotropic medications and need for help with ADL, which were significant in the baseline comparison. All $p$ values were two-sided and $\mathrm{p}$ values $<0.05$ were considered to be statistically significant.

\section{RESULTS}

Out of the 9957 cohort subjects, 9470 subjects (95\%) were followed between August 2008 and September 2009. The median duration of hospitalisation was 10.0 days (mean \pm SD $16.4 \pm 17.9$ ). The number of patients who fell during the follow-up period was $230(2.5 \%)$ and the incidence of falls was 3.28 per 100 person-days. The incidence of falls per 100 person-days by sex and age categories is shown in table 1 . In these patients, the median period from admission to the fall event was 30.0 days (mean \pm SD $20.6 \pm 22.0$ ).

The baseline characteristics of the subjects are shown in table 2. Factors associated with falls were compared

Table 2 Relationship between baseline characteristics and falls (\%), Fukushima Medical University Hospital cohort

\begin{tabular}{|c|c|c|c|}
\hline & \multicolumn{2}{|l|}{ Falls } & \multirow[b]{2}{*}{ p Value } \\
\hline & No & Yes & \\
\hline Number of patients & 9240 & 230 & \\
\hline Age (years) & $60.5 \pm 44.2$ & $68.0 \pm 16.4$ & $<0.05$ \\
\hline Men (\%) & 50.2 & 47.8 & 0.51 \\
\hline History of falls (\%) & 8.3 & 26.1 & $<0.05$ \\
\hline Cognitive dysfunction (\%) & 2.3 & 9.1 & $<0.05$ \\
\hline Planned surgery (\%) & 42.0 & 27.1 & $<0.05$ \\
\hline Wheelchair use (\%) & 19.0 & 48.7 & $<0.05$ \\
\hline Needs help to move (\%) & 13.5 & 41.3 & $<0.05$ \\
\hline Inhibition (\%) & 2.1 & 3.9 & 0.06 \\
\hline Use of a remote caring system (\%) & 0.7 & 3.9 & $<0.05$ \\
\hline Rehabilitation (\%) & 2.6 & 5.7 & $<0.05$ \\
\hline Laxative medication (\%) & 17.3 & 27.4 & $<0.05$ \\
\hline Sedative medication (\%) & 2.1 & 3.0 & 0.21 \\
\hline Hypnotic medication (\%) & 15.2 & 27.8 & $<0.05$ \\
\hline Psychotropic medication (\%) & 6.1 & 18.7 & $<0.05$ \\
\hline Needs help with ADL (\%) & 22.5 & 54.8 & $<0.05$ \\
\hline Impaired MMT (right foot) (\%) & 3.3 & 7.0 & $<0.05$ \\
\hline Impaired MMT (left foot) (\%) & 3.8 & 6.5 & 0.05 \\
\hline
\end{tabular}


Table 3 Association of baseline characteristics and falls in men, Fukushima Medical University Hospital cohort

\begin{tabular}{lllr}
\hline Baseline factors & OR & $\mathbf{9 5 \%}$ Cl & p Value \\
\hline Age & $\mathbf{1 . 0 2}$ & 1.01 to 1.04 & $<0.05$ \\
History of falls & $\mathbf{2 . 0 1}$ & 1.21 to 3.35 & $<0.05$ \\
Cognitive dysfunction & 0.87 & 0.41 to 1.85 & 0.73 \\
Planned surgery & 1.00 & 0.65 to 1.55 & 0.99 \\
Wheelchair use & 1.57 & 0.86 to 2.84 & 0.14 \\
Needs help to move & 1.75 & 0.95 to 3.23 & 0.07 \\
Use of a remote caring & 1.38 & 0.91 to 2.10 & 0.13 \\
system & & & \\
Rehabilitation & 0.50 & 0.20 to 1.25 & 0.14 \\
Laxative medication & 1.02 & 0.62 to 1.66 & 0.94 \\
Hypnotic medication & 1.06 & 0.61 to 1.84 & 0.84 \\
Psychotropic medication & $\mathbf{3 . 5 9}$ & 2.00 to 6.47 & $<0.05$ \\
Needs help with ADL & $\mathbf{2 . 2 8}$ & 1.38 to 3.78 & $<0.05$ \\
\hline Bold type indicates statistical significance. & \\
ADL, activities of daily living. & & & \\
\end{tabular}

between patients who do and did not fall during the follow-up period. No differences were seen in sex between the two groups, but there were significant differences in age, history of falling, cognitive dysfunction, planned surgery, wheelchair use, need for help to move, use of a remote caring system, rehabilitation, use of laxative, hypnotic or psychotropic medications, need for help with ADL and low MMT score (right foot only).

Tables 3 and 4 show the multivariable adjusted ORs of falls according to the sex of the patients. Age, history of falling and need for help with ADL were common risk factors for falls in both men and women. We found that age, history of falling and need for help with ADL were associated with an increased risk of falls (OR $1.02(95 \%$ CI 1.01 to 1.04 ), OR 2.01 (95\% CI 1.21 to 3.35$)$ and OR 2.28 (95\% CI 1.38 to 3.78$)$, respectively, for men and OR 1.02 (95\% CI 1.01 to 1.03$)$, OR 2.17 (95\% CI 1.38 to 3.40 ) and OR 1.74 (95\% CI 1.05 to 2.88 ), respectively,

Table 4 Association of baseline characteristics and falls in women, Fukushima Medical University Hospital cohort

\begin{tabular}{lllr}
\hline Baseline factors & OR & 95\% Cl & p Value \\
\hline Age & $\mathbf{1 . 0 2}$ & 1.01 to 1.03 & $<0.05$ \\
History of falls & $\mathbf{2 . 1 7}$ & 1.38 to 3.40 & $<0.05$ \\
Cognitive dysfunction & $\mathbf{2 . 5 6}$ & 1.24 to 5.26 & $<0.05$ \\
Planned surgery & $\mathbf{0 . 5 3}$ & 0.34 to 0.82 & $<0.05$ \\
Wheelchair use & 1.60 & 0.92 to 2.77 & 0.10 \\
Needs help to move & 1.58 & 0.89 to 2.80 & 0.12 \\
Use of a remote caring & 0.74 & 0.43 to 1.28 & 0.28 \\
system & & & \\
Rehabilitation & 1.28 & 0.86 to 1.91 & 0.22 \\
Laxative medication & 1.00 & 0.65 to 1.54 & 0.99 \\
Hypnotic medication & $\mathbf{1 . 6 5}$ & 1.07 to 2.56 & $<0.05$ \\
Psychotropic medication & 1.66 & 0.97 to 2.82 & 0.06 \\
Needs help with ADL & $\mathbf{1 . 7 4}$ & 1.05 to 2.88 & $<0.05$ \\
\hline Bold type indicates statistical significance. & & \\
ADL, activities of daily living. & & & \\
\end{tabular}

for women). Using psychotropic medication also increased the risk of falling in men (OR 3.59, 95\% CI 2.00 to 6.47 ), while cognitive dysfunction and use of hypnotic medication increased the risk of falling in women (OR 2.56, 95\% CI 1.24 to 5.26 and OR 1.65, 95\% CI 1.07 to 2.56 , respectively). Planned surgery was associated with a low risk of falls in women (OR 0.53, 95\% CI 0.34 to 0.82 ).

\section{DISCUSSION}

Multifactorial fall prevention programmes have recently been considered ${ }^{7-9}$ because falls are caused by complex risk factors such as disabilities and the caring environment. In the hospital setting it is very important to identify patients at a high risk for falling at admission to prevent the occurrence of falls. In the present study, age, a history of falling and the need for help with ADL were common risk factors for falls in both men and women, and it is suggested that it is important to obtain this information at admission. As falls are associated with restricted mobility, ${ }^{10}$ ADL are important for their prevention..

Medications such as psychotropic and hypnotic drugs increase the risk of falls. Some researchers have warned that multi-medication including antidepressants are a risk factor for falls. ${ }^{11}{ }^{12}$ Patients treated with psychotropic or hypnotic drugs therefore require special attention.

A retrospective study suggested that one of the independent risk factors for recurrent falls was a history of cognitive dysfunction, and cognitive impairment particularly affecting short-term memory, recall and visuospatial perception might contribute to recurrent falls in the inpatient population. ${ }^{13}$ In our prospective study, cognitive dysfunction increased the risk of falls in women. It is therefore necessary to monitor carefully the actions of patients with cognitive dysfunction.

Planned surgery was associated with a low risk of falling in women. The mean duration of hospitalisation was 16.4 days and the mean period between admission and falling was 20.6 days. With the use of a clinical path, the schedule of each inpatient should be properly planned which could lead to the prevention of falling.

It is important that doctors and medical coworkers carefully watch and evaluate patients with regard to their past history and present medications. This study did not consider disease types. It is important that more staff can evaluate easily the risk of falling.

In conclusion, it is very important to identify inpatients who are at a high risk of falling. Age, a history of falling and the need for help with ADL are the most important pieces of information to be taken at admission. Care plans that highlight fall prevention should be clear and considered, and patients should be monitored carefully when treated with psychotropic or hypnotic medications. Doctors and medical coworkers should carefully check the past history and present medications of patients. 
Author affiliations

${ }^{1}$ Department of Hygiene and Preventive Medicine, Fukushima Medical

University School of Medicine, Fukushima City, Japan

${ }^{2}$ Department of Clinical Quality Management, Fukushima Medical University Hospital, Fukushima City, Japan

${ }^{3}$ Department of Epidemiology and Public Health, Yokohama City University

Graduate School of Medicine, Yokohama City, Japan

Contributors TH conceived the idea of the study and was responsible for the design of the study and analysed the data. SH managed patients at admission and contributed to the interpretation of the result. NH, YK and TK participated in data collection. HK and TF participated in data analysis. All authors read and approved the report for submission.

Funding This study was supported by a health science research grant from Fukushima Medical University (Tokutei Research KPJ20002).

Competing interests None.

Patient consent Obtained

Ethics approval The study protocol was approved by the ethics board of Fukushima Medical University (approval registration No.726).

Provenance and peer review Not commissioned; externally peer reviewed.

Data sharing statement Extra data can be accessed via the Dryad data repository at http://datadryad.org/ with the doi:10.5061/dryad.16h8j.

Open Access This is an Open Access article distributed in accordance with the Creative Commons Attribution Non Commercial (CC BY-NC 4.0) license, which permits others to distribute, remix, adapt, build upon this work noncommercially, and license their derivative works on different terms, provided the original work is properly cited and the use is non-commercial. See: http:// creativecommons.org/licenses/by-nc/4.0/

\section{REFERENCES}

1. Kannus $P$, Sievänen $H$, Palvanen $M$, et al. Prevention of falls and consequent injuries in elderly people. Lancet 2005;366:1885-93.

2. American Geriatrics Society. Guideline for the prevention of falls in older persons. J Am Geriatr Soc 2001;49:664-72.

3. Leipzig RM, Cumming RG, Tinetti ME. Drugs and falls in older people: a systematic review and meta-analysis: II. Cardiac and analgesic drugs. J Am Geriatr Soc 1999;47:40-50.

4. Stevens JA, Phelan EA. Development of STEADI: a fall prevention resource for health care providers. Health Promot Pract 2013;14:706-14.

5. Karlsson MK, Vonschewelov T, Karlsson C, et al. Prevention of falls in the elderly: a review. Scand J Public Health 2013;41:442-54.

6. Bohannon R. Measuring muscle strength in neurological disorders. Fizyoterapi Rehabilitasyon 2005;16:120-33.

7. Gates S, Fisher JD, Cooke MW, et al. Multifactorial assessment and targeted intervention for preventing falls and injuries among older people in community and emergency care settings: systematic review and meta-analysis. BMJ 2008;336:130-3.

8. Tinetti ME, Baker DI, McAvay G, et al. A multifactorial intervention to reduce the risk of falling among elderly people living in the community. N Engl J Med 1994;331:821-7.

9. de Negreiros Cabral K, Perracini MR, Soares AT, et al. Effectiveness of a multifactorial falls prevention program in community-dwelling older people when compared to usual care: study protocol for a randomised controlled trial (Prevquedas Brazil). BMC Geriatr 2013;13:27.

10. Kosorok MR, Omenn GS, Diehr P, et al. Restricted activity days among older adults. Am J Public Health 1992;82:1263-7.

11. Tinetti ME. Preventing falls in elderly persons. $N$ Engl J Med 2003;348:42-9.

12. Marschollek M, Gövercin M, Rust S, et al. Mining geriatric assessment data for in-patient fall prediction models and high-risk subgroups. BMC Med Inform Decis Mak 2012;12:19.

13. Chen $X$, Van Nguyen $H$, Shen $Q$, et al. Characteristics associated with recurrent falls among the elderly within aged-care wards in a tertiary hospital: the effect of cognitive impairment. Arch Gerontol Geriatr 2011;53:e183-6. 